\title{
ANESTESIA PARA CESÁREA EN GESTANTE CON TETRALOGÍA DE FALLOT NO CORREGIDA
}

\author{
Ana Leslie Tijero Espinoza1, Dante Junior Segura Pinedo², Luis Enrique Sandoval Valiente ${ }^{3}$.
}

\begin{abstract}
RESUMEN
La Tetralogía de Fallot es la enfermedad cardíaca congénita cianótica más común. Los cambios hemodinámicos pueden producir complicaciones multiorgánicas graves y mortales, la elección de la anestesia es muy difícil. Las técnicas combinadas espinales epidurales se han convertido en una alternativa a las intervenciones neuroaxiales tradicionales; las ventajas del bloqueo subaracnoideo o espinal se fusionan con la flexibilidad de la técnica epidural; ofrecen un bloqueo espinal de instauración rápida, eficaz y de toxicidad mínima, mejora un bloqueo inadecuado y prolonga la duración de la anestesia con suplementos epidurales. El propósito de este artículo es reportar el caso de una gestante con diagnóstico de Tetralogía de Fallot no corregida y la técnica anestésica de eficaz instauración sin producir cambios hemodinámicos perjudiciales para el binomio madre-hijo.
\end{abstract}

Palabras claves: Técnica combinada espinal epidural; anestesia obstétrica; cesárea; Tetralogía de Fallot no corregida (Fuente: DeCS BIREME)

\section{ANESTHESIA FOR CAESAREAN SECTION IN PREGNANT WITH NON-CORRECTED FAILURE TETRALOGY}

\begin{abstract}
Tetralogy of Fallot is the most common cyanotic congenital heart disease. Hemodynamic changes can cause serious and fatal multiorgan complications, the choice of anesthesia is very difficult. Combined epidural spinal techniques have become an alternative to traditional neuroaxial interventions; the advantages of the subarachnoid or spinal block merge with the flexibility of the epidural technique; They offer a fast, effective and minimal toxicity spinal block, improves an inadequate block and prolongs the duration of anesthesia with epidural supplements. The purpose of this article is to report the case of a pregnant woman with a diagnosis of Tetralogy of Fallot uncorrected and the anesthetic technique of effective establishment without producing harmful hemodynamic changes for the mother-child binomial.
\end{abstract}

Keywords: Combined epidural spinal technique; obstetric anesthesia; Caesarean section; Tetralogy of Fallot not corrected (Source: MeSH NLM).

\section{INTRODUCCIÓN}

La Tetralogía de Fallot (TOF) es la forma más común de defecto cardíaco congénito cianótico, representa el 10\% de todos los casos, los continuos avances en técnicas quirúrgicas, estos pacientes ahora alcanzan la edad fértil y a menudo toleran el embarazo relativamente bien. Sin embargo, sin intervención quirúrgica, el $70 \%$ de las personas con TOF mueren en la primera década y solo alrededor del $3 \%$ sobrevive en la cuarta década. Por lo tanto, presentación de TOF no corregida en el embarazo es rara y presenta un riesgo significativo tanto para la madre como para el neonato ${ }^{1}$.
Se han publicado varios modelos de estratificación de riesgo: la puntuación de riesgo CARPREG (enfermedad de CARdiac en PREGnancy), la puntuación de riesgo ZAHARA (Zwangerschap bij Aangeboren HARtA fwijkingen I) clasificación modificada de la OMS (mWHO) y la escala New York Heart Association (NYHA $)^{2}$. Una desviación importante de la anestesia general hacia la regional primordialmente en países desarrollados se ha vinculado a un decremento en la mortalidad relacionada con la anestesia. La Anestesia Combinada Epidural Espinal (ACEE) tiene grandes ventajas en la embarazada en cirugía para cesárea como trabajo de parto. La técnica ACEE

Médico Residente del Departamento de Anestesia Analgesia y Reanimación. Universidad Nacional Federico Villarreal. Lima- Perú.

Médico Anestesiólogo del Departamento de Anestesia Analgesia y Reanimación del INMP, Fellowship en Anestesia Obstétrica por la World Federation

Societies of Anesthesiologists en la Clínica Bolivariana en Medellin Colombia, Instructor DDAMOS, Miembro de la Sociedad Peruana de Dolor y Cuidados Paliativos. Lima-Perú.

3 Médico Jefe del Servicio de Centro Quirúrgico del Departamento de Anestesia Analgesia y Reanimación, Miembro de la Sociedad Peruana de Anestesia Analgesia y Reanimación. Lima-Perú.

Citar como: Tijero AL, Segura DJ, Sandoval L. Anestesia para Cesárea en gestante con Tetralogia de Fallot no Corregida. Rev Peru Investig Matern

Perinat. 2019;8(4): 59-61

DOI https://doi.org/10.33421/inmp.2019176

Recibido: 10-10-2019 Aceptado: 12-12-2019 
ofrece un bloqueo espinal de instauración rápida, eficaz y de toxicidad mínima, prolonga la duración de la anestesia con suplementos epidurales ${ }^{3}$.

Existen varias formas de llevar a cabo el bloqueo ACEE, sus diferencias radican fundamentalmente en la punción en uno o dos espacios con una o dos agujas. La elección del acceso a un único espacio probablemente se acompañe de menor incidencia de cefalea, punción venosa epidural, hematoma, infección y dificultad técnica si lo comparamos con el acceso a dos espacios. Aunque el bloqueo a dos espacios conlleva menos riesgo de producir un bloqueo subaracnoideo extendido o total, debido a la mayor distancia entre el agujero de la duramadre y la punta del catéter ${ }^{4}$

El presente Reporte de Caso tiene como objetivo dar a conocer el manejo de un caso de paciente gestante con Tetralogía de Fallot no corregida y determinar que Técnica Anestésica resulta segura en cirugía para cesárea, con ello aportar información de utilidad para el manejo anestésico.

\section{REPORTE DEL CASO}

Primigesta de 29 años, de 37 semanas de gestación, antecedente de TOF no corregido, acude por emergencia en trabajo de parto fase activa, disnea leve a moderada.

Evaluación pre anestésica: IMC de $20.65 \mathrm{~kg} / \mathrm{m}^{2}$, ASA III, CARPREG > 1, FC 76/min., FR 22/min., PA 122/63 $\mathrm{mmHg}$. SO $295 \%, \mathrm{FiO}_{2} 21 \%$, apertura oral $3 \mathrm{~cm}$., DTM $>6$ cm., Mallampati III, FCF 144/min., RQ III, cianosis perioral y lecho ungüeal, dedos de manos y pies en palillo de tambor (Fig. 01).

Ap. Cardiovascular: Se ausculto segundo ruido único, fuerte con soplo sistólico foco pulmonar IV/VI.

Ap. Respiratorio: Murmullo vesicular pasa bien en ambos campos pulmonares, no estertores.

\section{Exámenes de laboratorio:}

\begin{tabular}{|c|c|c|c|c|}
\hline $\begin{array}{c}\text { Hb 13.2 } \\
\text { g/dL }\end{array}$ & TP 13.4" & $\begin{array}{c}\text { Plaquetas } \\
283000\end{array}$ & $\begin{array}{c}\text { Glucosa } \\
73 \mathrm{mg} / \mathrm{dL}\end{array}$ & $\begin{array}{c}\text { Urea } 17 \\
\mathrm{mg} / \mathrm{dL}\end{array}$ \\
\hline Hto 40\% & TTP 38.6" & $\begin{array}{c}\text { Fibrinógeno } \\
389 \mathrm{mg} / \mathrm{dL}\end{array}$ & $\begin{array}{c}\text { Creatinina } \\
0.65 \mathrm{mg} / \\
\mathrm{dL}\end{array}$ & $\begin{array}{c}\text { Proteínas } \\
6.20 \\
\mathrm{~g} / \mathrm{dL}\end{array}$ \\
\hline
\end{tabular}

Ecocardiograma: Del tercer trimestre.

\begin{tabular}{|c|c|c|c|c|}
\hline $\begin{array}{c}\text { Fracción } \\
\text { de eyección } \\
65 \%\end{array}$ & $\begin{array}{c}\text { Tetralogía } \\
\text { de Fallot }\end{array}$ & $\begin{array}{c}\text { Función } \\
\text { sistólica } \\
\text { biventricular } \\
\text { conservada }\end{array}$ & $\begin{array}{c}\text { Derrame } \\
\text { pericárdico } \\
\text { moderado }\end{array}$ & $\begin{array}{c}\text { Cabalgamient } \\
\text { o de aorta }\end{array}$ \\
\hline $\begin{array}{c}\text { Estenosis } \\
\text { pulmonar }\end{array}$ & $\begin{array}{c}\text { Disfunción } \\
\text { diastólica } \\
\text { severa }\end{array}$ & $\begin{array}{c}\text { Crecimiento } \\
\text { de la aurícula } \\
\text { derecha } \\
\text { severa }\end{array}$ & $\begin{array}{c}\text { Hipertrofia } \\
\text { ventricular } \\
\text { derecha }\end{array}$ & CIV $6 \mathrm{~mm}$. \\
\hline
\end{tabular}

\section{Técnica anestésica}

Paciente en DLI (Fig. 02) se infiltra piel con lidocaína al $2 \%$ en espacio L3-L4, se inserta trocar de Touhy \#18, con pérdida de resistencia hasta llegar al espacio epidural, se inserta el trocar espinal punta lápiz \# 27, se administra Bupivacaína hiperbárica 5 mg. al 0,5\%, Fentanilo 10 ug., Morfina 100 ug.,_se inserta catéter epidural cefálico a 12 $\mathrm{cm}$., paciente en decúbito dorsal se administra Lidocaína $100 \mathrm{mg}$. al $2 \%$ sin epinefrina por catéter epidural; con la ACEE aguja tras aguja se logra un nivel anestésico adecuado, se da inicio a la cirugía, se obtiene un producto de $2950 \mathrm{~kg}$, con apgar $8^{1} 9^{5}$, con restricción de volúmenes, con perfusión de $\mathrm{CINa} 0.9 \%$ B.H.E (-), durante el intraoperatorio hemodinámica se mantuvo estable con mínimos cambios, postoperatorio en $\mathrm{UCl}$ intermedios por 24 horas, alta del servicio.

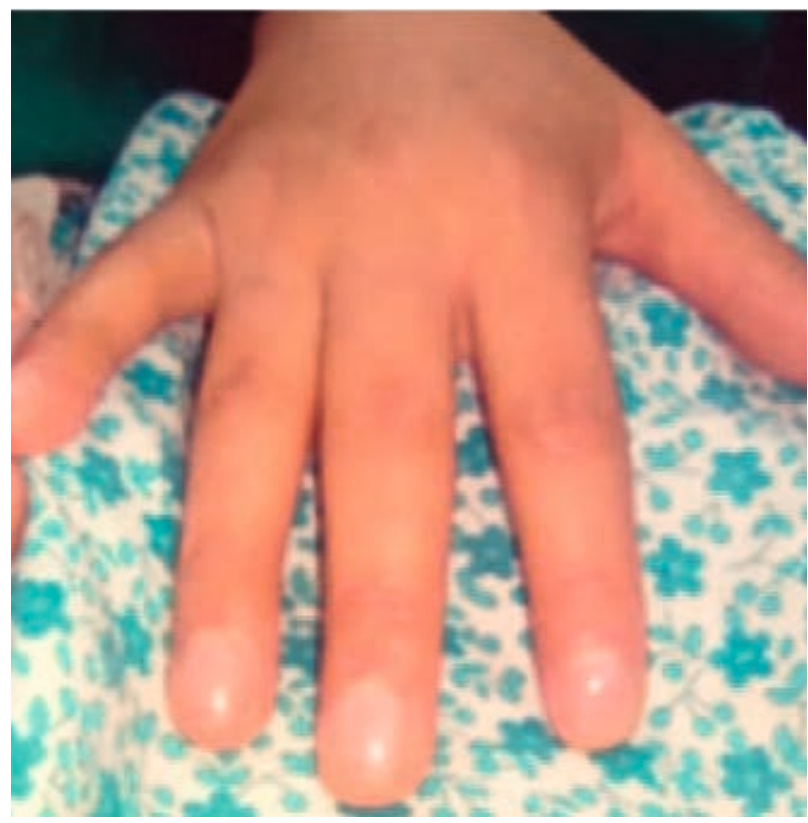

Figura 1

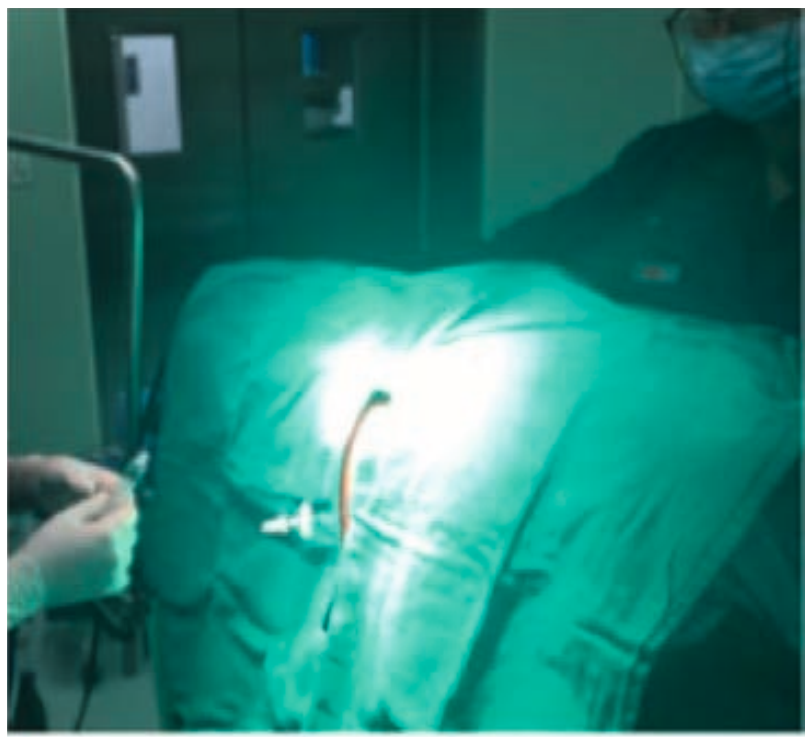

Figura 2 


\section{DISCUSIÓN}

La enfermedad cardíaca está emergiendo como la causa indirecta más importante de la muerte materna a nivel mundial, pueden estar enmascaradas por una mayor carga de volumen, o ser causadas por el embarazo. Los datos de mortalidad contextualizan el riesgo de enfermedad cardíaca en el embarazo con tasas de letalidad cercanas a 1 en 200, esto comparado a otras complicaciones obstétricas que se consideran de alto riesgo.

Se estratifica a la gestante NYHA clase III o IV con tasa de eventos del $27 \%$ pronosticada, tomando en cuenta el estado de la gestante con TOF no corregido, estenosis pulmonar, disfunción diastólica severa.

La técnica ACEE, se emplea en la práctica obstétrica en pacientes con cardiopatías para proporcionar analgesia y anestesia de calidad y eficacia, mediante el empleo de varias combinaciones de fármacos, reduciendo los requerimientos de agentes anestésicos empleados, con mínimos cambios hemodinámicos a pesar de los cambios fisiológicos propios per se y el bloqueo a dosis menores del anestésico local espinal reduce el riesgo de bloqueo espinal alto e hipotensión materna ${ }^{5}$.

La ventaja de esta técnica aguja a través de la aguja con la paciente en posición decúbito lateral izquierdo, evita la compresión aorto-cava, el catéter epidural se utiliza para complementar el bloqueo espinal previo mapeo en la paciente y para la analgesia postoperatoria.

Aunque los medicamentos atraviesan la placenta y pueden medirse en el neonato, los anestésicos locales no causan depresión neonatal. ${ }^{6}$

En conclusión, la ACEE resultó ser una técnica segura para cesárea en esta paciente con TOF no corregido, con bloqueo sensitivo y motor adecuado, con cambios hemodinámicos mínimos; no agravó condición cardiovascular preexistente, con una mejor recuperación y manejo del dolor postoperatorio.

\section{RECOMENDACIONES}

Evaluación pre anestésica días previos a la cirugía para el manejo perioperatorio.

Uso profiláctico de vasopresores evitando cambios hemodinámicos mayores.

No sobrecarga hídrica y uso de catéter epidural para analgesia posoperatoria.

\section{AGRADECIMIENTOS}

A todas las personas que hacen posible la realización de este reporte de caso.

Financiamiento: Autofinaciado.

Conflicto de interés: Los autores declaran no tener ningún conflicto de intereses

\section{REFERENCIAS BIBLIOGRÁFICAS}

1. Drenthen W, Boersma E, Balci A, et al. Predictors of pregnancy complications in women with congenital heart disease. Eur Heart J 2010; 31:2124-32.

2. Van Hagen IM, Boersma E, Johnson MR, et al. Global cardiac risk assessment in the Registry of Pregnancy and Cardiac disease. Eur J Heart Fail. 2016; 18:523-33.

3. Van de Velde M. Combined spinal epidural analgesia for labor and delivery: a review. Acta Anaesthesiol Belg 2004; 55: 177.

4. Hughes D, Simmons S W, Brown J, Cyna M. Combined spinalepidural. Cochrane Database Syst Rev 2003; 4: CD003401.

5. Ngan Kee WD. The use of vasopressors during spinal anaesthesia for caesarean section. Curr Opin Anaesthesiol. 2017; 30:319-325.

6. Littleford J. Effects on the fetus and newborn of maternal analgesia and anesthesia: a review. Can J Anaesth. 2004; 51:586-609.

\section{Correspondencia:}

Ana Leslie Tijero Espinoza.

Dirección: Jr. Miroquesada 941, Cercado de Lima-Perú.

Correo electrónico: dr.juanpablo_108@hotmail.com

Teléfono: 916676794 